\title{
Natural history of cardiovascular manifestations in Marfan syndrome
}

\author{
C D M van Karnebeek, M S J Naeff, B J M Mulder, R C M Hennekam, M Offringa
}

\begin{abstract}
Aims-To investigate the natural history of mitral valve and aortic abnormalities in patients with Marfan syndrome during childhood and adolescence.

Methods-Fifty two patients with Marfan syndrome were followed for a mean of 7.9 years. Occurrence of adverse cardiovascular outcomes was measured clinically and by ultrasound examination.

Results-Mitral valve prolapse (MVP) was diagnosed in 46 patients at a mean age of 9.7 years, more than $80 \%$ of whom presented as "silent MVP". Mitral regurgitation (MR) occurred in 25 patients, aortic dilatation in 43 , and aortic regurgitation (AR) in 13. Both MVP and aortic dilatation developed at a constant rate during the age period 5-20 years. In 23 patients MVP was diagnosed before aortic dilatation, in 18 the reverse occurred, and in 11 patients the two abnormalities were diagnosed simultaneously. During follow up, 21 patients showed progression of mitral valve dysfunction; progression of aortic abnormalities occurred in 13. Aortic surgery was performed in 10; two died of subsequent complications. Mitral valve surgery was performed in six. In sporadic female Marfan patients the age at initial diagnosis of MVP, MR, aortic dilatation, and $A R$ was lowest, the grade of $M R$ and AR most severe, the time lapse between the occurrence of MVP and subsequent MR as well as between dilatation and subsequent AR shortest, and the risk for cardiovascular associated morbidity and mortality highest.

Conclusions-During childhood and adolescence in Marfan syndrome, mitral valve dysfunction as well as aortic abnormalities develop and progress gradually, often without symptoms, but may cause considerable morbidity and mortality by the end of the second decade, especially in female sporadic patients. (Arch Dis Child 2001;84:129-137)
\end{abstract}

Keywords: Marfan syndrome; cohort study; mitral valve; aorta

Marfan syndrome is an autosomal dominantly inherited disorder of connective tissue with pleiotropic manifestations exerted by $F B N-1$ gene mutations in the classic triad of ocular, skeletal, and cardiovascular systems. ${ }^{1-3}$

Anomalies of the cardiovascular system account for a significant proportion of the shortened life span of patients with Marfan syndrome. ${ }^{4}$ As the progressive dilatation of the aortic root with its propensity towards aortic regurgitation, dissection, or rupture is the most common life threatening feature of Marfan syndrome, clinical investigation has focused primarily on this particular complication. ${ }^{67}$ Although mitral valve dysfunction is a frequent finding in children with Marfan syndrome and has been suggested to be of prognostic significance for further cardiovascular complications, especially if accompanied by valvular insufficiency, it has received much less attention..$^{8-11}$

We report on the natural history of these two cardiovascular manifestations, and associated complications, in Marfan patients from childhood to early adulthood.

\section{Methods}

PATIENT SELECTION, DIAGNOSIS, AND FOLLOW UP We searched the data files of the paediatric department at our institution to identify all individuals who had been evaluated for Marfan syndrome and related connective tissue disorders between October 1981 and April 1997. Of the 223 individuals identified, 68 patients were diagnosed with Marfan syndrome based on the diagnostic criteria proposed by Beighton et al. ${ }^{12}$ The diagnosis of Marfan syndrome in all patients was recently reconfirmed by a single clinical geneticist ( $\mathrm{RH})$ using the adapted criteria by DePaepe et al. ${ }^{13}$ Standard procedures included complete clinical, cardiac, and ophthalmologic investigation of the patient as well as both parents. Our study cohort is comprised of 52 of these 68 patients with Marfan syndrome, who met the following inclusion criteria: non-neonatal phenotype; age less than 16 years at initial evaluation; at least two echocardiograms performed before the age of 16 years; and serial follow up data of at least one year. We excluded the three patients with neonatal Marfan syndrome as their clinical course is distinct and would influence results to a large extent.

Follow up of each of these patients is defined as the time interval between first and most recent visit.

\section{DATA COLLECTION}

We reviewed all medical records for the following: gender; age at diagnosis; and presence of Marfan syndrome and/or aortic dissection in first and second degree relatives. For each clinical and echocardiographic examination during follow up, data were collected on anthropometric measurements, cardiovascular findings (described below), use of beta blockers, cardiac surgery, and hospitalisations. 
CARDIAC EXAMINATION

Cardiac examinations of all patients under the age of 17 years were performed by a single paediatric cardiologist; those older than 17 by a single adult cardiologist. For the present study, only data concerning auscultation and echocardiography were used. Cardiac auscultation was performed in the supine and left lateral decubitus positions before, during, and after the Valsalva manoeuvre. Murmurs were graded at the maximal intensity by a standard I to VI system.

\section{ECHOCARDIOGRAPHY}

Echocardiograms were performed with standard equipment using $3.5,5$, or $7.5 \mathrm{mHz}$ transducers for paediatric patients (depending on body size) and $2.5 \mathrm{mHz}$ transducers for patients above the age of 17 years.

The function of the mitral valve was assessed on transthoracic two dimensional standard echocardiographic images, most relevant of which were the parasternal short and long axis views and the apical two and four chamber views. Patients were instructed to lie in either the supine or the left lateral decubitus position, depending on optimal visualisation of both anterior and posterior leaflets. Presence of the various cardiac abnormalities was assessed applying the criteria listed below.

DEFINITIONS

Mitral valve prolapse (MVP)

On the parasternal long axis view an end systolic moderate to severe bowing of the anterior and/or posterior mitral valve leaflet(s) into the left atrium with coaptation of the leaflets at the level of, or on the atrial side of the mitral annulus. ${ }^{14}$

Mitral regurgitation (MR)

Assessed during systole on the parasternal short and long axis views as well as the apical two and four chamber views, using the pulsed Doppler technique until 1986, and afterwards by colour flow Doppler. Grading was based on a semiquantitative technique of characterising Doppler echocardiography: grade 1, trivial to mild; grade 2 , moderate; grade 3 , severe. ${ }^{15}$

Mitral valve dysfunction

The presence of either MVP or MR (grade 1 or more) based on the above definitions.

Progression of mitral valve dysfunction

(1) Appearance of MR in a patient with a previous diagnosis of MVP; (2) appearance of MVP in a patient with a previous diagnosis of $M R$; (3) an increase in the grade of $M R$ in a patient with a previous positive finding on echocardiography; (4) an increase in the grade of MR necessitating surgery; or (5) causing death.

\section{Aortic dilatation}

A diameter of the aortic root on the parasternal long axis view exceeding the expected mean (based on a nomogram normalising for body surface area) ${ }^{16}$ by more than two standard deviations. ${ }^{17}$
Aortic regurgitation (AR)

Assessed during diastole on the parasternal long axis view as well as the apical or subcostal five chamber view. Severity was graded using the method described for MR: grade 1, trivial to mild; grade 2, moderate; and grade 3, severe. ${ }^{15}$

\section{Aortic abnormalities}

Presence of aortic dilatation and/or AR (grade 1 or more) based on the above definitions.

\section{Progression of aortic abnormalities}

(1) Appearance of AR in a patient with a previous diagnosis of aortic dilatation; (2) increase in the grade of $A R$ in a patient with a previous positive finding on echocardiography; (3) aortic surgery because of progressive dilatation with or without AR; (4) death related to aortic complications.

\section{Morbidity and mortality}

The occurrence in a patient of one or more of the following events: mitral valve surgery; aortic surgery; aortic dissection; or death.

\section{STATISTICAL ANALYSIS}

We estimated the MVP free, MR free, aortic dilatation free, and AR free survival using the Kaplan-Meier product limit method. ${ }^{18}$ We evaluated these probabilities as a function of attained age since birth and the risk factors gender and family history. Univariate statistics and Kaplan-Meier product limit estimators were calculated with the SAS-PC statistical package (SAS Institute Inc., Cary, North Carolina).

\section{Results}

PATIENT CHARACTERISTICS

The study group comprised 25 male and 27 female patients with Marfan syndrome. Table 1 presents the clinical characteristics. There were no significant differences in these characteristics between males and females. Thirty three individuals had a positive family history for Marfan syndrome; 20 were probands and 13 were siblings. Of these familial cases, 26 $(78.8 \%)$ had at least one family member who had had an aortic dissection. The number of echocardiograms per patient ranged from two to 31 (mode 12 ). Six patients $(12 \%)$ used beta blockers for one year or longer.

Atrial septal defect of the secundum type was detected in eight patients (seven females, one male) and a bicuspid aortic valve in one male.

Table 1 Clinical characteristics $(n=52)$

\begin{tabular}{lcl}
\hline & Mean & Range \\
\hline Age at initial evaluation (y) & 7.9 & $1.0-16$ \\
Age at most recent evaluation (y) & 15.5 & $3.0-28$ \\
Length of follow up (y) & 7.7 & $2.0-15$ \\
& No. (\%) & Male \\
Familial Marfan cases (\%) & $33(63)$ & 15 \\
Sporadic Marfan cases (\%) & $19(37)$ & 10 \\
Pectus deformity (\%) & $35(67)$ & 14 \\
$\quad$ Excavatum (\%) & $13(25)$ & 8 \\
Carinatum (\%) & $22(42)$ & 13 \\
Scoliosis (\%) & $23(44)$ & 12 \\
Ectopia lentis (\%) & $32(62)$ & 14 \\
\hline
\end{tabular}


Table 2 Auscultatory and echocardiographic findings of mitral valve prolapse on initial evaluation

\begin{tabular}{ccc}
\hline & $\begin{array}{l}\text { All pts (\%) } \\
(n=52)\end{array}$ & $\begin{array}{l}\text { Male (\%) } \\
(n=25)\end{array}$ \\
\hline Concordance & $28(54)$ & $12(43)$ \\
click-/echo- & $23(44)$ & $10(44)$ \\
click+/echo+ & $5(10)$ & $2(40)$ \\
Disconcordance & $24(46)$ & $13(54)$ \\
click-/echo+ & $22(42)$ & $12(55)$ \\
click+/echo- & $2(4)$ & $1(50)$ \\
\hline
\end{tabular}

AUSCULTATION VERSUS ECHOCARDIOGRAPHY

For all patients, the echocardiographic and auscultatory data concerning MVP at initial evaluation were compared (table 2). In 28 cases $(54 \%)$ these two methods agreed on the presence or absence of an abnormal finding (concordance). Of the 26 patients in our cohort with MVP on the initial echocardiogram, five cases could be diagnosed on auscultation, implying that more than $75 \%$ have "silent" MVP. Silent MVP occurred with equal frequency in males (42\%) and females $(55 \%)$.

MVP AND MR

MVP was diagnosed in 46 patients (88\%); mean age at initial diagnosis was 9.7 years (range 1.0 to 18.2 years, fig $1 \mathrm{~A}$ ). The KaplanMeier curve shows a steady decline from the age of 4 years until 18 years, after which no patient developed MVP.

MR was diagnosed in 25 patients (48\%), mean age 12.2 years (range 1.0 to 24.3 ); severity at initial diagnosis was assessed as mild in all (fig 2A). The Kaplan-Meier curve shows a more or less regular decline, associated with an unchanging hazard of MR.

AORTIC DILATATION AND AR

Aortic dilatation was diagnosed in 43 patients ( $83 \%$; fig 3A), six of whom were subsequently prescribed beta blockers. The Kaplan-Meier curve shows a steady decline until the age of 16 years (with a somewhat steep phase between age 4 and 7 years), after which only one patient developed dilatation until age 25 years.

AR was diagnosed in 13 patients (25\%; fig 4A). The severity of AR at initial diagnosis was mild (grade I) in 12 patients and moderate (grade II) in one. Mean age at initial diagnosis was 14.6 years (range 5.3 to 25.7 years). AR did not occur below 5 years of age; thereafter, the Kaplan-Meier curve declines regularly.
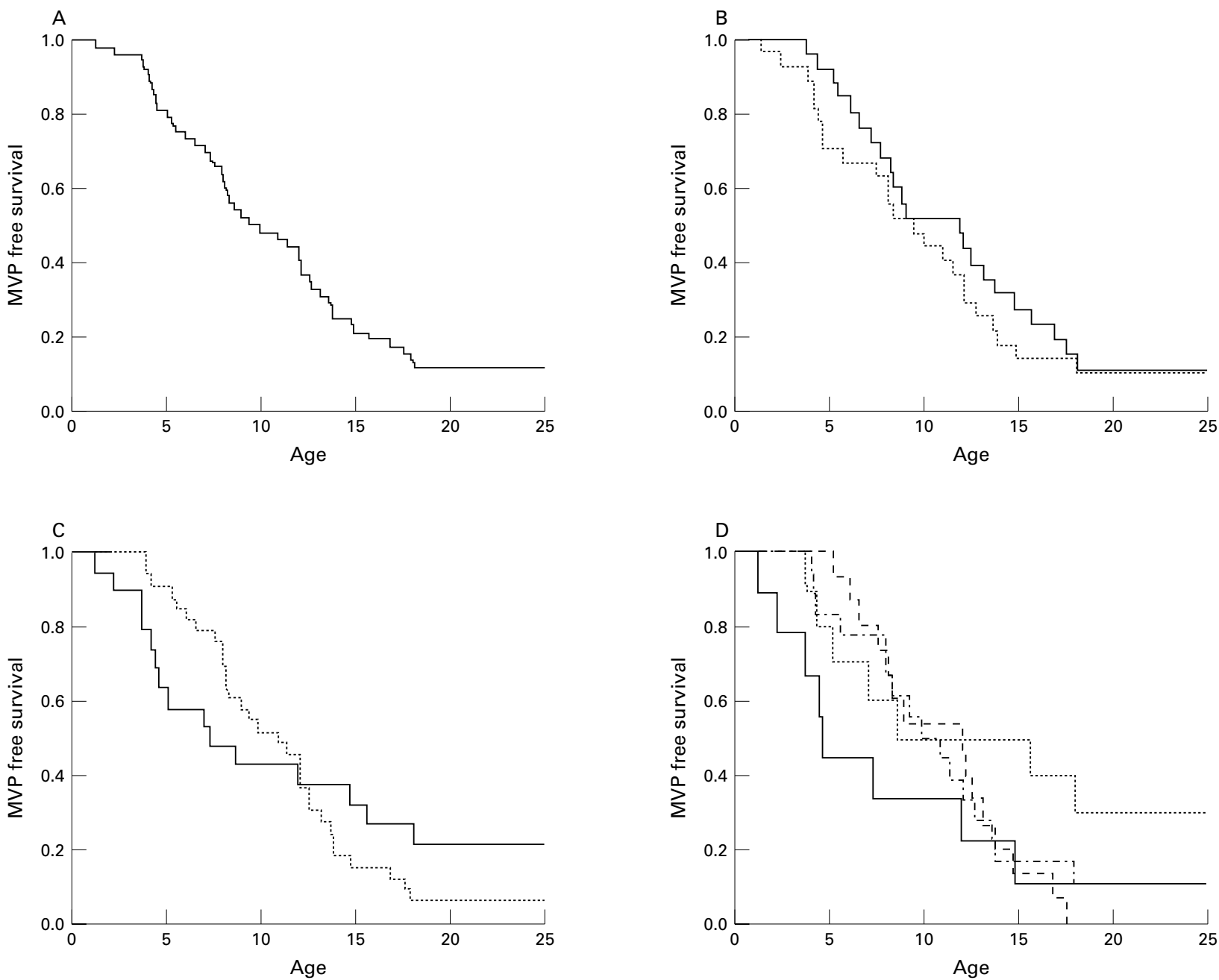

Figure 1 Mitral valve prolapse (MVP) free survival since birth. $(A)$ Total cohort $(n=52)$; (B) cohort by gender: males ( $n=25$, straight line) and females ( $n=27$, dotted line); $(C)$ cohort by presence of a positive family history for Marfan syndrome: sporadic cases ( $n=19$, straight line) and familial cases ( $n=33$, dotted line); (D) cohort by gender and family history: male familial cases (long hatched line), male sporadic cases (dotted line), female familial cases (short hatched line), female sporadic cases (straight line). Each step in the curve denotes the diagnosis of MVP in a patient. 
RELATION OF MITRAL DYSFUNCTION AND AORTIC ABNORMALITIES WITH OTHER CLINICAL

CHARACTERISTICS

Gender

Twenty two of 46 patients (48\%) who developed MVP were male (fig 1B). Throughout the age interval from birth to 18 years, MVP tended to manifest earlier in females than in males (statistically not significant). The probability of MR was higher in females (50\%) compared to males $(40 \%, \mathrm{p}<0.05$; fig $2 \mathrm{~B})$; MR manifested earlier in females compared to males, comparable with the findings of MVP.

Twenty two of the 43 patients (53\%) with aortic dilatation were male (fig 3B). During the interval from birth to 10 years, dilatation occurred earlier in females. No difference in AR probability was found between males $(54 \%)$ and females (46\%), nor for the mean age at AR occurrence (fig 4B).

\section{Family history}

Thirty one patients $(67 \%)$ with MVP had a positive family history for Marfan syndrome. Before the age of 12 years, MVP was diagnosed earlier in sporadic patients, but thereafter earlier in familial cases (fig 1C). MR developed in familial cases $(n=14)$ at a constant rate and before 13 years in $44 \%$ of the sporadic cases (fig 2C).The frequency of aortic dilatation was similar in the familial and the sporadic group (fig 3C). The overall frequency of AR was similar among familial and sporadic cases, but all sporadic cases developed AR before the age of 13 years (fig 4C), whereas AR did not develop before 16 years in familial cases.

\section{Gender and family history}

Based on the combination of characteristics "gender" and "family history", the frequency of MVP was as follows: 15/15 (patients with $\mathrm{MVP} /$ total number of patients in group) in the male familial group, $7 / 10$ in the male sporadic group; $16 / 18$ in the female familial group, and $8 / 9$ in the female sporadic group (fig 1D). The age at initial diagnosis of MVP was lowest in the female sporadic group $(\mathrm{p}<0.05)$.

The frequencies of MR in these four groups were $6 / 15$ in the male familial group, $4 / 10$ in the male sporadic cases, $9 / 18$ in the female familial group, 6/9 in the female sporadic group (fig 2D). As with MVP, age at initial diagnosis of MR was the lowest in sporadic females.
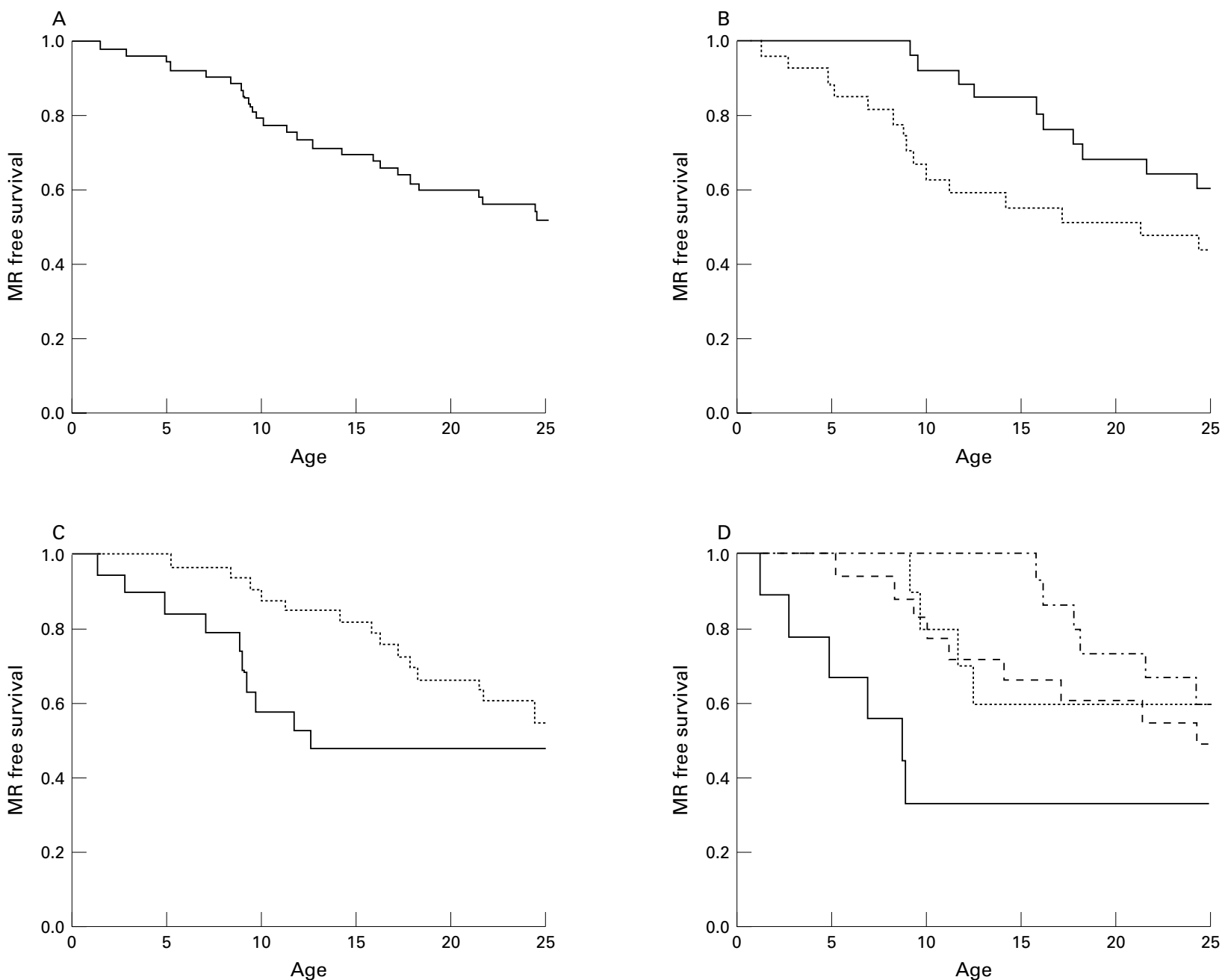

Figure 2 Mitral regurgitation (MR) free survival since birth. (A) Total cohort $(n=52)$; (B) cohort by gender: males $(n=25$, straight line) and females ( $n=27$, dotted line); (C) cohort by presence of a positive family history for Marfan syndrome: sporadic cases $(n=19$, straight line) and familial cases ( $n=33$, dotted line); (D) cohort by gender and family history: male familial cases (long hatched line), male sporadic cases (dotted line), female familial cases (short hatched line), female sporadic cases (straight line). Each step in the curve denotes the diagnosis of mitral regurgitation in a patient. 
Frequencies of aortic dilatation were 15/15 in the male familial group, $8 / 10$ in the male sporadic group, $13 / 18$ in the female familial group, and $7 / 9$ in the female sporadic group.

Occurrence of $A R$ in the same groups was $4 / 15$ in the male familial group, 3/10 in the male sporadic group, $2 / 17$ in the female familial group, and $4 / 10$ in the female sporadic group. Sporadic patients, both female and male, were younger at the time of initial diagnosis of AR.

TIME RELATION BETWEEN INITIAL DIAGNOSIS OF MVP AND AORTIC DILATATION

In 23 patients (44\%), MVP was diagnosed before aortic dilatation. Eight of these patients did not develop dilatation during the study period. The time interval between diagnosis of MVP and dilatation for the remaining 15 patients averaged 8.5 months (range 6.3 to $113.9)$. In 18 patients (35\%) aortic dilatation was diagnosed before MVP. Of these 18 patients, three had not developed MVP in the study period. For the remaining 15 patients, the mean time interval between the diagnosis of the two cardiac abnormalities was 22 months (range 11.7 to 88.1 ). For the remaining
11 patients $(21 \%)$ the initial diagnosis of MVP and aortic dilatation was simultaneously made on the same echocardiogram.

PROGRESSION OF MV DYSFUNCTION

During follow up 21 patients (40\%; nine male, 13 familial) showed progression of mitral valve dysfunction. In 18, MVP progressed to MR (interval mean 4.6 years, range 0.5 to 10.4 years); in three patients MR was the primary diagnosis and MVP was diagnosed later. Progression in severity of MR was seen in six cases: five were sporadic females who progressed from a mild to moderate grade of MR, and one was a sporadic male who progressed from a mild to a severe grade. Mitral valve surgery was performed in two cases with progressive $M R$.

No relation was found between the age at MVP presentation and the interval lapse to MR. However, when grouping the patients by gender and family history, it was found that in sporadic female patients age at initial diagnosis of MVP was relatively low and the time interval to further progression of MR was shorter.
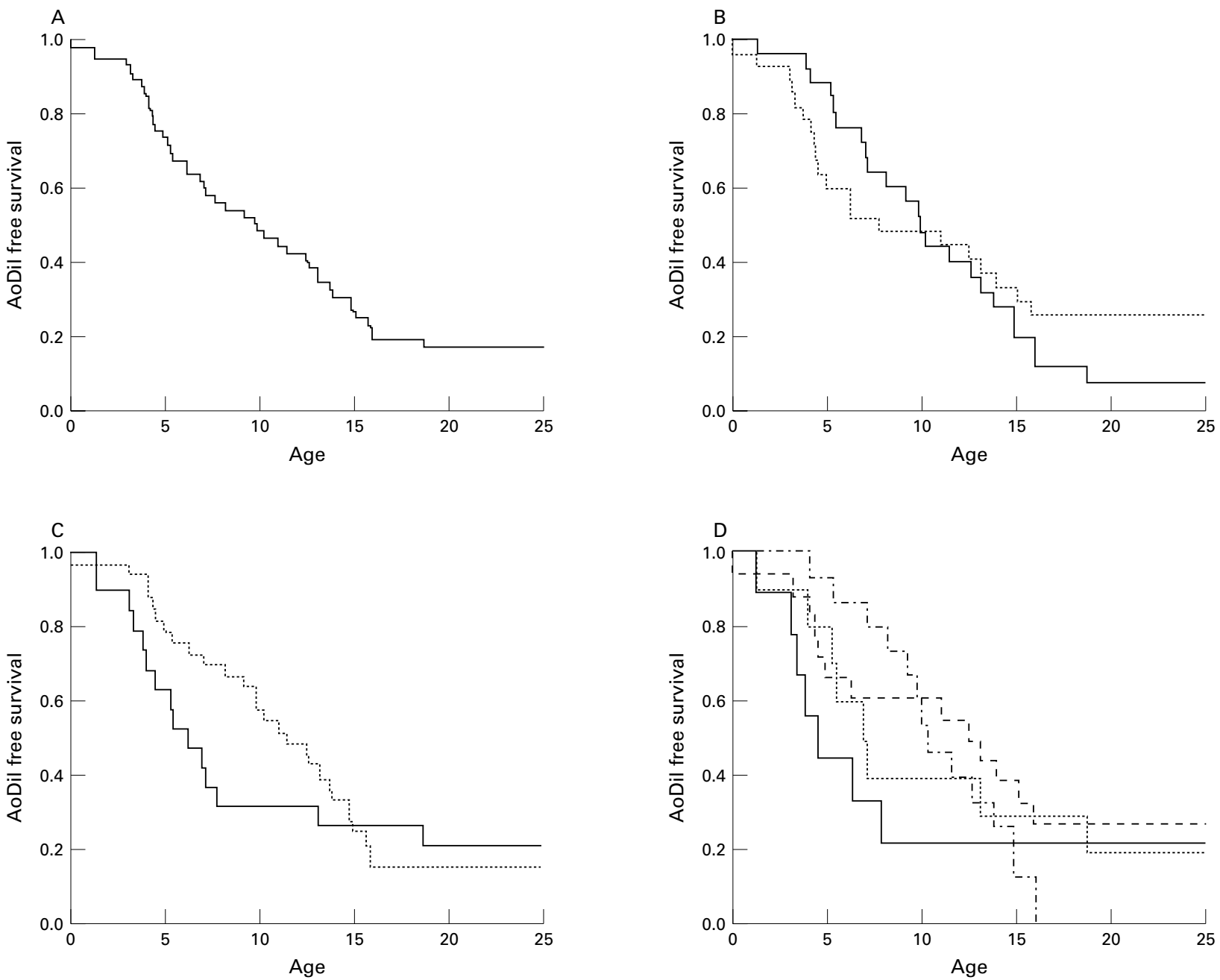

Figure 3 Aortic dilatation (AoDil) free survival since birth. (A) Total cohort $(n=52)$; (B) cohort by gender: males ( $n=25$, straight line) and females $(n=27$, dotted line); $(C)$ cohort by presence of a positive family history for Marfan syndrome: sporadic cases $(n=19$, straight line) and familial cases ( $n=33$, dotted line); (D) cohort by gender and family history: male familial cases (long hatched line), male sporadic cases (dotted line), female familial cases (short hatched line), female sporadic cases (straight line). Each step in the curve denotes the diagnosis of aortic dilatation in a patient. 
PROGRESSION OF AORTIC ABNORMALITIES

During follow up 12 patients (25\%; six male; six familial) showed progression of aortic abnormalities, four of whom were sporadic females (interval mean 5.1 years, range 0 to 12.1). No relation was found between the interval and the age at development of aortic dilatation. For sporadic female patients the age at initial diagnosis of dilatation was low and the time interval to further progression of $\mathrm{AR}$ was short.

Four patients showed progression in AR severity: one (female, sporadic) progressed from mild to moderate grade, and three (one familial female, two familial males) from mild to severe grade. All 10 patients who underwent aortic surgery had progression of aortic dysfunction.

MORTALITY AND MORBIDITY

In the study period, 10 patients $(19 \%)$ eventually required cardiovascular surgery. Two died after initial evaluation: one (female, sporadic) died shortly after aortic root replacement at age 12.3 years from ventricular fibrillation, the other (female, familial) died at age 21.4 years in the postoperative period as a result of inoper- able redissection of the aorta near the end patch of the graft located at the left subclavian artery.

Eight patients underwent replacement of a severely dilated aortic root; two of these required acute operation for aortic dissection. On the last preoperative echocardiogram, two other patients had moderate to severe aortic insufficiency. The postoperative follow up was as follows: three years after initial surgery one patient required the placement of a new prosthetic aortic valve for rapidly increasing aortic insufficiency; another underwent aortic reoperation for repair of suture dehiscence near the end patch 0.6 years postoperatively. In a third patient the aorta dissected at the level of the left subclavian artery and soon afterwards also at the level of the renal arteries; acute surgery was performed succesfully in both instances. In the other five patients with a history of cardiovascular surgery, no major aortic complications occurred during postoperative follow up.

Mitral valve surgery was performed in six patients, all with moderate to severe MR (three female sporadic; one sporadic male; two familial male). In five patients who underwent repair
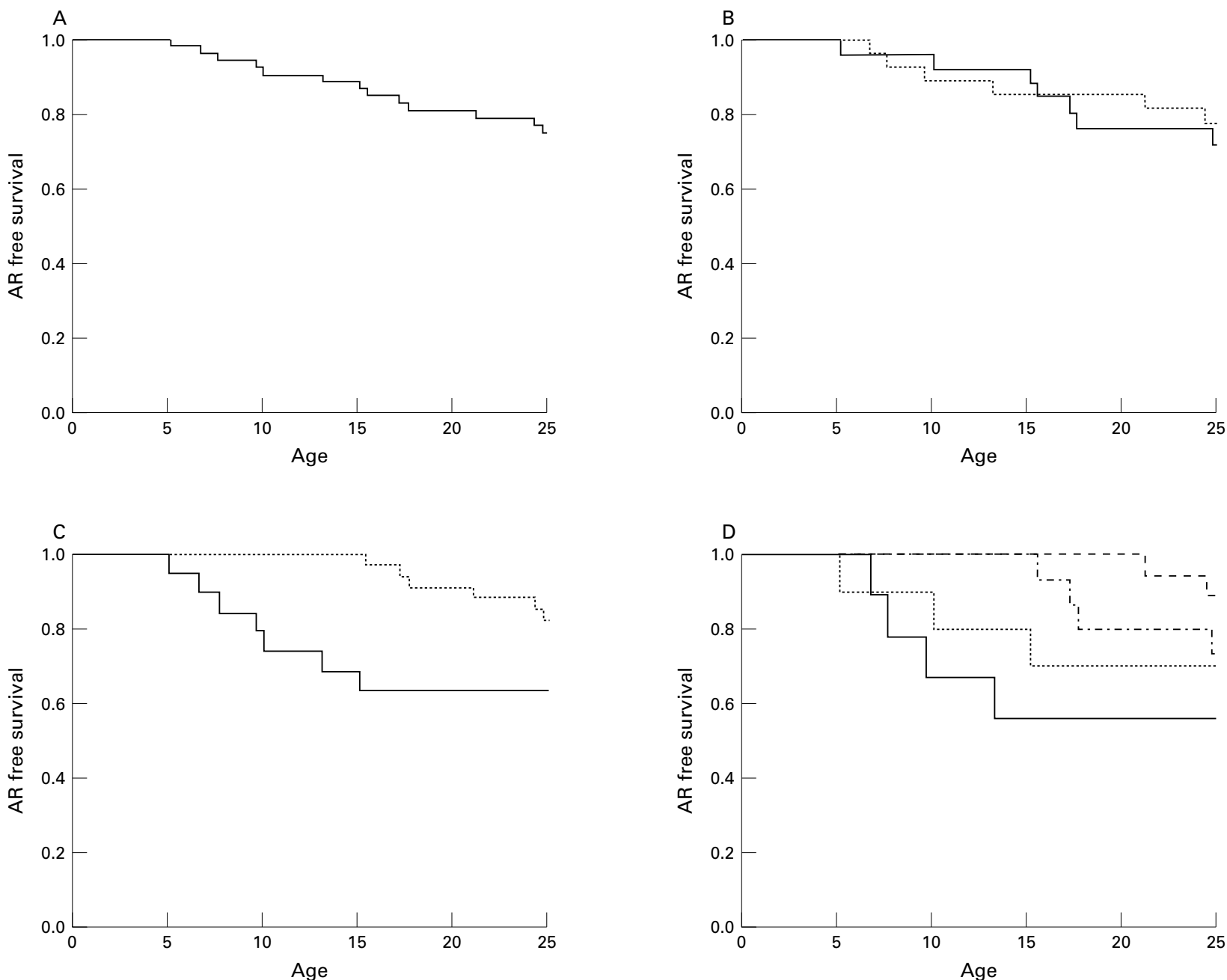

Figure 4 Aortic regurgitation (AR) free survival since birth. (A) Total cohort $(n=52) ;(B)$ cohort by gender: males $(n=25$, straight line) and females ( $n=27$, dotted line); (C) cohort by presence of a positive family history for Marfan syndrome: sporadic cases $(n=19$, straight line) and familial cases ( $n=33$, dotted line); (D) cohort by gender and family history: male familial cases (long hatched line), male sporadic cases (dotted line), female familial cases (short hatched line), female sporadic cases (straight line). Each step in the curve denotes the diagnosis of aortic regurgitation in a patient. 
with a mitral annuloplasty; the procedure was concomitant to aortic surgery. In one of these, a sporadic female, extensive calcification of the mitral valve and left atrial wall was identified during surgery. Replacement with a prosthetic valve was performed in one male patient at age 23 years, after a rapidly progressive detoriation of MV dysfunction resulting in an unexpected rupture of the chordae tendinae. On postoperative follow up all patients showed a slightly abnormal valve, three trivial $\mathrm{MR}$, and three mild MR.

No bacterial endocarditis occurred during the study period. The protocol in our hospital prescribes antibiotic prophylaxis against subacute bacterial endocarditis in all Marfan patients with mitral valve dysfunction and/or AR.

\section{Discussion}

The most prominent cardiovascular manifestations of Marfan syndrome, MVP and aortic dilatation, are now known to be caused by defects in fibrillin 1, the structural protein of these cardiac tissues. MVP in Marfan syndrome is thought to develop by means of the following: fibromyxomatous changes in leaflets and chordae tendineae, calcification of the annulus, abnormal annular distensibility, and possibly mitral valve enlargement. ${ }^{19}$ These abnormalities may result in the billowing or prolapse of anterior and/or posterior mitral valve leaflets with herniation and redundancy into the corresponding atrium, followed by mitral regurgitation and in the worst case rupture of the chordae tendineae. ${ }^{8}{ }^{10}$ Defects in the fibrillin 1 protein also result in a progressive aneurysmal dilatation of the ascending aorta. Although the entire aortic wall is weakened, dilatation usually occurs at the sinuses of Valsalvae. As the weakened aortic root progressively dilates, the leaflets of the aortic wall fail to close completely and regurgitation occurs.

Although the $F B N-1$ gene encoding for fibrillin has been identified, the diagnosis of Marfan syndrome is still firmly based on clinical evaluation of abnormalities in different organ systems and family history. ${ }^{12}{ }^{13}$ As manifestations are age dependent, progressive, and vary extensively both between and within families, diagnosis and management are often difficult, necessitating long term and regular follow up, especially in children. ${ }^{52021}$ The present study aimed to increase the understanding of the role of age and selected clinical features in the occurrence of cardiovascular complications.

STRENGTHS AND WEAKNESSES OF THE STUDY

The retrospective design of this study and the nature of Marfan syndrome itself precludes an accurate definition of the age at which cardiac abnormalities actually occur in these patients. Only the age at first detection can be used, which will be higher than the true age at occurrence in a solitary case. This problem is inherent to a progressive disorder such as Marfan syndrome: most of the clinical features indicat- ing a patient to be affected develop during life, and the diagnosis can only be made retrospectively.

In adult Marfan patients, prophylactic beta blockade has been shown to be effective in slowing the rate of aortic dilatation and reducing the risk of aortic complications. ${ }^{22}$ However, because of a lack of similar studies in younger patients and the risk of side effects at a young age, paediatric Marfan patients in our hospital are not routinely prescribed beta blockers. Therefore the present study is a description of the natural history of cardiovascular manifestations, especially aortic dilatation.

The relatively large number of paediatric and adolescent Marfan patients in this cohort, sporadic and familial alike, as well as the long and complete follow up period, constitute strengths of this study of the natural history of mitral valve dysfunction in relation to gender and family history. As all familial and sporadic patients were studied in the same way and were followed over time by a small group of investigators without loss to follow up, despite the limitations, a number of conclusions may be drawn.

AUSCULTATION VERSUS ECHOCARDIOGRAPHY A substantial proportion of our patients with MVP on two dimensional echocardiogram lacked auscultatory signs and presented as silent MVP. This finding concurs with previous studies of adult Marfan patients, ${ }^{823-25}$ in which the reported echocardiographic evidence of MVP (with or without MR) ranged from $78 \%$ to $100 \%$, largely exceeding the estimated prevalence based on auscultatory evidence, which varied from $45 \%$ to $74 \% .^{82326} 27$ Therefore, we recommend that all paediatric and adolescent patients suspected for Marfan syndrome be evaluated echocardiographically for the presence of MVP.

PREVALENCE AND AGE AT OCCURRENCE OF MV DYSFUNCTION AND AORTIC ABNORMALITIES

During childhood and adolescence, patients with Marfan syndrome are already at a high risk of developing MVP. In other studies MVP prevalence varied from $35 \%$ to $100 \%,{ }^{2528}$ concurring with a prevalence in our patient cohort of $88 \%$. During follow up MR developed in $48 \%$, comparable to frequencies in paediatric cohorts of other Marfan studies with ranges from $44 \%$ to $58 \% .{ }^{9}{ }^{29} \mathrm{MR}$ is associated with considerable complications, and its presence at such a young age negatively influences overall prognosis.

Aortic dilatation was present in four of every five patients in our cohort, a finding comparable to that of other studies. ${ }^{21}{ }^{28-30}$ AR occurred in $25 \%$; other studies report prevalences of AR varying between $15 \%$ and $44 \%{ }^{28}{ }^{29}$ As AR may be seen as a complication of aortic dilatation strongly associated with life threatening cardiovascular events, such as rupture, it is important to note that during childhood and adolescence it may develop in one of every four Marfan patients.

In the present study, the chance of diagnosing MVP or aortic dilatation increased from the 
age of 5 years onwards, more or less linearly into early adolescence for both cardiac abnormalities. For example, at age 5, the chance of finding MVP in a Marfan patient was about $17 \%$, while at age 15 this chance had increased to $75 \%$. The risk for MR also increased linearly with age.

In our cohort all patients developed MVP before the age of 18 years, all but one developed dilatation before 16 , and more than half developed $\mathrm{MR}$ and one quarter AR before age 25 . Therefore, childhood and adolescence can be considered the crucial period in Marfan syndrome for development of cardiovascular abnormalities. Within this early period, it is not possible to identify a specific age at which the risk for developing these abnormalities is particularly high. Regular echocardiographic controls are mandatory throughout the entire period, and in patients with only minor or no cardiac abnormalities on initial echocardiograms.

ORDER OF APPEARANCE OF THE MAJOR CARDIAC MANIFESTATIONS

As mitral dysfunction is the primary cause of morbidity and mortality in infants, ${ }^{31}$ it has been assumed that children are mostly affected by mitral dysfunction, while in adults dilatation of the aorta is more common. ${ }^{27}{ }^{32}$ This suggests that mitral dysfunction develops earlier during life. However, the results of our study indicate that the number of patients that develop mitral dysfunction before aortic dysfunction is similar to the percentage with first an aortic and then a mitral dysfunction.

\section{RISK FACTORS ASSOCIATED WITH}

CARDIOVASCULAR ABNORMALITIES

The cumulative incidence of MVP was equal for males and females, which has also been reported by others. ${ }^{8329}$ However, MR as a complication of MVP was more common among females than males, and the age of initial diagnosis of MVP and MR was lower in females. Thus, during childhood and adolescence females seem to be at a higher risk for early development of mitral dysfunction. No such relation was found between aortic abnormalities and gender.

It has been observed that sporadic cases of Marfan syndrome, especially with the neonatal phenotype, have more severe cardiovascular involvement compared to familial cases. ${ }^{53}$ In our study group, in which patients with the neonatal type were excluded, this remains unclear: the cumulative incidence of MV dysfunction and aortic abnormalities was lower in sporadic cases, but the age of first detection was higher in familial cases. Sporadic Marfan patients may be at a higher risk of developing cardiovascular abnormalities more early in life, but further studies are needed in this respect.

When combining the role of gender and family history, the studied numbers in each group become small, but it does seem that sporadic female patients may be at a higher risk of developing complications of MVP and aortic dilatation at a younger age. Thus, we suggest that female patients with a negative family his- tory be monitored very closely for cardiac dysfunction.

\section{MORTALITY AND MORBIDITY}

In the last decades the life expectancy of Marfan patients has increased from 37 years as estimated in 1972 to more than 61 years in 1995, the latter recently reconfirmed by a study performed in 1998. ${ }^{34-36}$ This increase has been attributed to advances in aortic surgery and medical treatment, a larger proportion of patients diagnosed with a milder form of the disease, and an overall increase in life expectancy in the general population.

Mortality in our cohort was $6 \%$ up to the age of 25 years, which is low in comparison to rates reported in other studies (range 10$22 \%){ }^{2127} 28$ In childhood and adolescence, abnormalities of the aorta are foremost responsible for surgical intervention and associated mortality. ${ }^{37-39}$ Our results are quite similar: by 25 years of age, aortic dysfunction requiring surgery was present in one of five patients. AR was present in all patients necessitating surgery, and was especially severe in the two patients who died. Development of AR in a young patient should lead to intensified follow up and treatment.

\section{CONCLUSIONS}

Based on this study and the current literature we conclude that during childhood and adolescence in Marfan syndrome:

(1) Mitral valve dysfunction often presents as "silent MVP", and may develop and progress without symptoms: it is therefore important to evaluate all Marfan patients echocardiographically, even those with minor or no cardiovascular abnormalities on initial evaluations.

(2) MVP and aortic dilatation are common, with a similar cumulative incidence, developing at a similar constant rate throughout this age period and without a specific order in their appearance.

(3) $\mathrm{MR}$ and $\mathrm{AR}$ are less common, with $\mathrm{MR}$ occurring more frequently and earlier in life than AR. AR is an indicator of high risk for subsequent complications such as dissection.

(4) Morbidity and mortality are associated with aortic abnormalities rather than with mitral valve dysfunction.

(5) Female sporadic cases seem to be most likely to develop both mitral valve dysfunction and aortic abnormalities and their complications early in life.

1 Magenis RE, Maslen CL, Smith L, Allen L, Sakai LY. Localisation of the fibrillin (FBN) gene to chromosome 15, band q21.1. Genomics 1991;11:346-51.

2 Dietz HC, Cutting GR, Pyeritz RE, et al. Marfan syndrome caused by a recurrent de novo missense mutation in the fibrillin gene. Nature 1991;352:337-9.

3 Kainulainen K, Pulkkinen L, Savoilainen A, Kaitila I, Peltonen L. Location on chromosome 15 of the gene defect causing Marfan syndrome. N Engl f Med 1990;323:935-9.

4 Pyeritz RE. Disorders of fibrillins and microfibrilogenesis: Marfan syndrome, MASS phenotype, contractural arachnMarfan syndrome, MASS phenotype, contractural arachnodactyly and related conditions. In: Emery AE, Rimoin DL, David L, Connor JM, Pyeritz RE, eds. Principles and practice of medical genetics, 3rd edn. New York: Churchill 5 Pyeritz RE, McKusick VA. The Marfan syndrome: diagnosis and management. N Engl F Med 1979;300:722-7. 
6 Roman MJ, Rosen SS, Kramer-Fox R, Devereux RB. The prognostic significance of the pattern of aortic root dilatation in the $M$

7 Legget ME, Unger TA, O'Sullivan CK, et al. Aortic root complications in Marfan's syndrome: identification of a lower risk group. Heart 1996;75:389-95.

8 Pyeritz RE, Wappel MA. Mitral valve dysfunction in the Marfan syndrome. Clinical and echocardiographic study of prevalence and natural history. Am f Med 1983;74:797807.

9 Geva T, Hegesh J, Fraud M. The clinical course and echocardiographic features of Marfan syndrome in childhood. Am F Dis Child 1987;141:1179-82.

10 Geva T, Sanders SP, Diogenes MS, Rockenmacher S, Van Praagh R. Two-dimensional and Doppler echocardiographic and pathologic characteristics of the infantile Marfan syndrome. Am f Cardiol 1990;65:1230-7.

11 Gillinov AM, Zehr KJ, Redmond JM, et al. Cardiac operations in children with Marfan's syndrome: indications operations in children with Marfan's syndrom

12 Beighton P, De Paepe A, Danks D, et al. International nosology for heritable disorders of connective tissue, Berlin nosology for heritable disorders of connectiv

13 DePaepe A, Devereux RB, Dietz HC, Hennekam RCM, Pyeritz RE. Revised diagnostic criteria for the Marfan syndrome. Am f Med Genet 1996;62:417-26.

14 Krivokapich J, Child JS, Dadourian BJ, Perloff JK. Reassessment of echocardiographic criteria for diagnosis of mitra valve prolapse. Am $\mathcal{F}$ Cardiol 1988;61:131-5.

15 Miyatake K, Okamoto M, Kinoshita N, et al. Semi quantitative grading of severity of mitral regurgitation by real-time two-dimensional Doppler flow imaging technique. $7 A m$ Coll Cardiol 1986;7:82-8.

16 Haycock GB, Schwartz GJ, Wisotsky DH. Geometric method for mearuring body surface area: a height-weight formula validated in infants, children and adults. $\mathcal{F}$ Pediatr 1978;93:62-6.

17 Roman MJ, Devereux RB, Kramer-Fox R, O'Loughlin J. Two-dimensional echocardiographic aortic root dimen-
sions in normal children and adults. Am 7 Cardiol 1989;64: 507-12.

18 Kaplan EL, Meier P. Nonparametric estimation from incomplete observations. F Am Stat Assoc 1958;53:203-23.

19 Pini R, Roman MJ, Kramer-Fox R, Devereux RB. Mitra valve dimensions and motion in Marfan patients with and without mitral valve prolapse: comparison to primary mitral valve prolapse and normal subjects. Circulation 1989;80:915-24.

20 Lacro RV. Marfan syndrome. In: Burg FD, Ingelfinger JR, Wald ER, Polin RA, eds. Gellis and Kagan's current paediatric therapy 15. Philadelphia: WB Saunders Co., 1996: 171-3.

21 Lipscomb KJ, Caylton-Smith J, Harris R. Evolving phenotype of Marfan's syndrome. Arch Dis Child 1997;7:41-6.

22 Shores J, Berger KR, Murphy EA, Pyeritz RE. Progression of aortic dilatation and the benefit of long-term betaadrenergic blockade in Marfan's syndrome. N Engl f Med 1994;330:1335-41.
23 Brown OR, DeMots H, Kloster FE, Roberts A, Menashe VD, Beals RK. Aortic root dilatation and mitral valve pro-
lapse in Marfan's syndrome: an echocardiographic study. lapse in Marfan's syndrom

24 Jeresaty RM, Landry AB Jr, Liss JP. Silent mitral valve prolapse: analysis of 32 cases. Am $\mathcal{F}$ Cardiol 1975;35:146.

25 Roberts WC. Congenital cardiovascular abnormalities usually silent until adulthood: morphologic features of the floppy mitral valve, valvular aortic stenosis, discrete subvalvular aortic stenosis, hypertrophic cardiomyopathy, sinus of Valsalva aneurysm, and the Marfan syndrome. Cardiovasc Clin 1979;10:407.

26 McKusick VA. The cardiovascular aspects of Marfan's syndrome: a heritable disorder of connective tissue. Circulation 1955;11:321-42.

27 Marsalese DL, Moodie DS, Vacante M, et al. Marfan's syndrome: natural history and long-term follow-up of cardiovascular involvement. F Am Coll Cardiol 1989;14: $422-8$.

28 Geva T, Hegesh J, Fraud M. The clinical course and chocardiographic features of Marfan syndrome in childhood. Am f Dis Child 1987;141:1179-82.

29 Van Karnebeek CDM, Gauvreau K, Colan SD, Lacro RV. Clinical and echocardiographic predictors of adverse cardiovascular outcome in childhood Marfan syndrome [abstract]. 7 Am Coll Cardiol 1998;31:70a-71a.

30 Fleischer KJ, Nousari HC, Anhalt GJ, Stone CD, Laschinger JC. Immunohistochemical abnormalities of Laschinger JC. Immunohistochemical abnormalities of Ann Thorac Surg 1997;63:1012-17.

31 Geva T, Sanders SP, Diogenes MS, Rockenmacher S, Van Praagh R. Two-dimensional and Doppler echocardiographic and pathologic characteristics of the infantile Marfan syndrome. Am f Cardiol 1990;65:1230-7.

32 Brown OR, DeMots H, Kloster FE, Roberts A, Menashe VD, Beals RK. Aortic root dilatation and mitral valve prolapse in Marfan's syndrome: an echocardiographic study. Circulation 1975;53:651-7.

33 Pan CW, Chen CC, Wang SP, Hsu TL, Chiang BN. Echocardiographic study of cardiac abnormalities in families of patients with Marfan syndrome. F Am Coll Cardiol 1985;6:1016-20.

34 Murdoch JL, Walker BA, Halpern BL, Kuzma JW, McKusick VA. Life expectancy and causes of death in Marfan syndrome. N Engl f Med 1972;286:804-8.

35 Silverman DI, Burton KJ, Gray J, et al. Life expectancy in the Marfan syndrome. Am f Cardiol 1995;75:157-60.

36 Gray JR, Bridges $\mathrm{AB}$, West RR, et al. Life expectancy in British Marfan syndrome populations. Clin Genet 1998;54: $124-8$.

37 Gillinov AM, Zehr KJ, Redmond JM, et al. Cardiac operations in children with Marfan's syndrome: indications and results. Ann Thorac Surg 1997;64:1140-5.

38 Silverman DI, Burton KJ, Gray J, et al. Life expectancy in the Marfan syndrome. Am $\mathcal{F}$ Cardiol 1995;75:157-60.

39 Tsang VT, Pawade A, Karl TR, Mee RBB. Surgical management of Marfan syndrome in children. $\mathcal{F}$ Card Surg 1994;9:50-4. 